\title{
Interactive sensemaking in Authorship networks
}

\author{
Bram Vandeputte \\ Dept. Computerwetenschappen \\ K.U.Leuven \\ Celestijnenlaan 200A \\ 3000 Leuven, Belgium \\ bram.vandeputte@cs.kuleuven.be
}

\author{
Erik Duval \\ Dept. Computerwetenschappen \\ K.U.Leuven \\ Celestijnenlaan 200A \\ 3000 Leuven, Belgium \\ erik.duval@cs.kuleuven.be
}

\author{
Joris Klerkx \\ Dept. Computerwetenschappen \\ K.U.Leuven \\ Celestijnenlaan 200A \\ 3000 Leuven, Belgium \\ joris.klerkx@cs.kuleuven.be
}

\begin{abstract}
This paper describes research on rich opportunities for novel interaction on large multitouch tables to assist researchers. We have designed, developed and evaluated ResearchTable which provides an interactive visualization of (co-)authorship networks. Our evaluation shows that users discovered relevant researchers and papers that they were unaware of.
\end{abstract}

ACM Classification: H.5.2Information Interfaces and PresentationUser Interfaces[Evaluation/methodology]

\section{General terms: Design,Experimentation}

Keywords: publication networks, sensemaking, multitouch, information visualization

\section{Introduction}

Exploring publications is an important part of what a researcher does. New interfaces are needed to facilitate this [4]. It is vital to use a suitable representation for exploring a network of (publication) data [8]. We choose to visualize the publication network. Combining this with multitouch makes the exploration experience more profound [3].

In this paper, we describe the development and evaluation of Researchtable, a new interactive tabletop visualization tool.

\section{Researchtable}

To visualize the search space, there are plenty of opportunities. We could include (co-)authorship relations, forward and backward citations [1], geospatial information about affiliations [6], topical relationships based on concept extraction [5], etc.

We focus on authorship, as it "carries" trust and relevance. The authorship network is visualized using a node-link diagram, where authors and papers form the nodes, and authorship relationships are the edges. Using a node-link diagram renders relations explicit and allows for visual navigation across the network [3], which plays a key role in exploratory search. When visualizing the (co-)authorship network, high profile authors can be used as landmarks [11][7]. Therefor,

Permission to make digital or hard copies of all or part of this work for personal or classroom use is granted without fee provided that copies are not made or distributed for profit or commercial advantage and that copies bear this notice and the full citation on the first page. To copy otherwise, to republish, to post on servers or to redistribute to lists, requires prior specific permission and/or a fee.

Conference ITS'11, November 13-16, Kobe, Japan.

Copyright 2011 ACM 978-1-4503-0871-7/11/11...\$10.00. we make the font size of the author name proportional to the number of publications, so that prolific authors will become landmarks through their larger size. Two implementation and evaluation cycles have been completed. The next sections explain for each iteration its goals and outcomes. We evaluated the Researchtable at two conferences.

\section{First exploration}

The goals of the first iteration were to gather preliminary feedback from researchers on usefulness and usability. The app shows the complete graph lay-out. A spring-graph algorithm with physical forces updates the graph continuously as a user interacts with the visualization. The advantage is that the network stays organized. However, when too many nodes continue to move for longer periods of time, this becomes problematic when users build their mental model. Interaction with the application is possible through singletap, long-tap, drag, pan, pinch and rotate gestures. Filter and search functionalities are also available.

The evaluation was done at EC-TEL 2010. We have collected metadata of 332 full papers and 818 authors, making up 1148 authorship relations spread over 5 editions of the conference. The participants where given tasks to find the most prolific authors, to search for authors related to themselves and to discover related researchers. In total, eleven participants filled in feedback forms. Based on these, we can conclude that ResearchTable is fun to use and useful to understand the structure of a research domain. $63 \%$ of the participants found unknown relevant researchers and papers. They could easily find themselves, and they found new people in their surroundings. The users also investigated the bigger network structure and reasoned about the most prolific authors in the network. However the participants found it harder to find indirectly related authors. This indicates that navigation through the network could be further improved. There were also useful suggestions for extension: automatically calculating and highlighting the shortest path between authors, visualizing the evolution of a network over time and using topic information for the network layout.

\section{Overview First vs Start from what you know}

The feedback from the first iteration triggered our interest in how researchers change their exploratory process based on the overview technique. We therefor decided to compare two well known HCI visualization techniques. The first technique follows Ben Shneiderman's Mantra "Overview first, zoom and filter, then details-on-demand" [10], which we will 


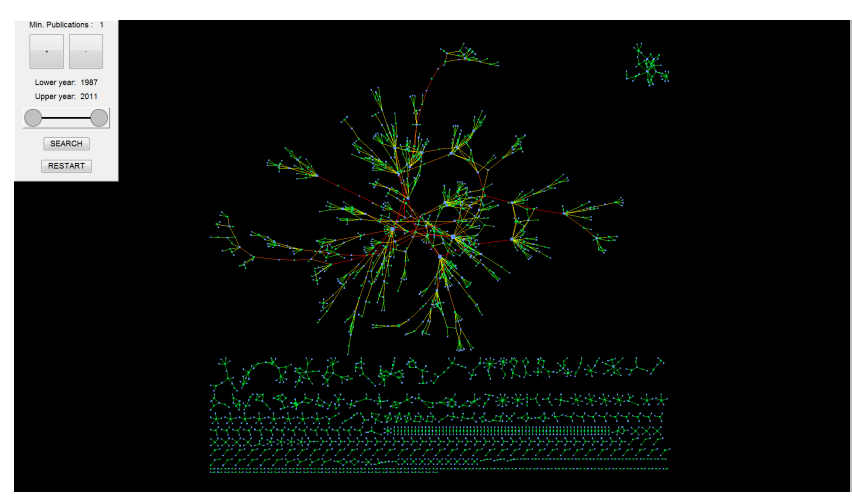

Figure 1: The "Overview first" application

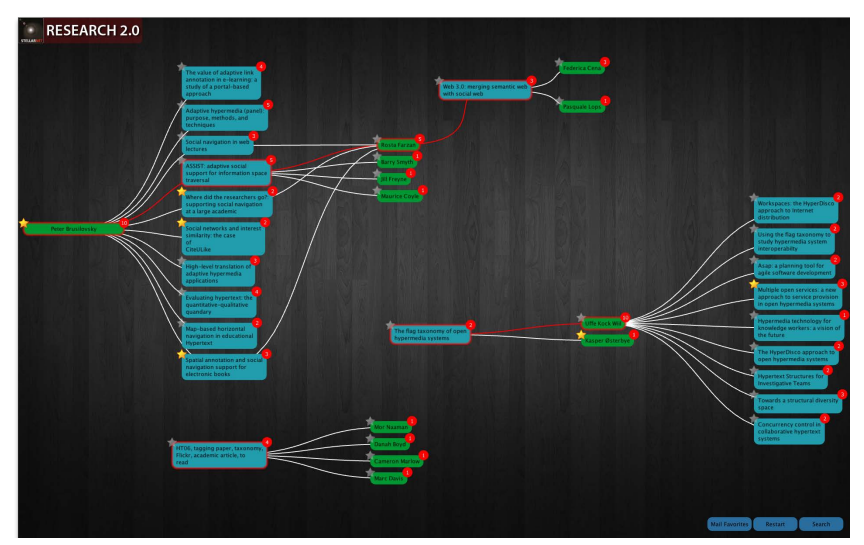

Figure 2: The "Start from what you know" app

call overview first. The second technique is based on Jeffrey Heer's "Start from what you know, then grow" [2]. To compare these techniques we implemented two new versions of Researchtable, and evaluated how researchers discovered interesting authors and papers in both versions. This evaluation was done at Hypertext 2011, with 17 participants using both applications for the same amount of time. The data was scraped from the ACM digital library website. 934 papers were gathered with 1601 different authors, making up 2440 authorship relations. Figure 1 illustrates the overview first application. It is based on Cytoscape [9], as the first version of Researchtable had scalability and lay-outing issues, and Cytoscape features highly scalable network visualization and has build in graph layout options. Starting with a view on the complete network, it allows the user to search, pan, zoom and filter to explore the network.

The start from what you know application, shown in Figure 2 , is a modified version of the first iteration. It now starts with an empty screen, forcing the user to start with a search. When a user clicks on search results, they appear on the screen as nodes of a network. The network can be explored by expanding the nodes and exposing direct neighbors or by performing additional searches. First evaluation results show that only 7 out of 17 participants used filtering. Four used it regularly (more than once). This low use could be explained by assuming that most people do not have a high need for filtering, as they can pan and zoom around to "filter down" on the things they find interesting. The pan and zoom functionalities were used more intensively in the overview first application, while in the start from what you know application people kept on a detailed zoom level during their whole exploration phase.

Overall, the results suggest that the participants were taking a different approach in their sensemaking process in both apps. The overview first application was used more for getting acquainted with the overall structure of the network, and thus looking for known or prolific authors to start their voyage. In the start from what you know application, people where more interested in getting to know who was working on a particular topic and what they were working on.

\section{Conclusion}

This work searches to gain insight in interactive sensemaking of authorship networks. A first evaluation shows that ResearchTable is fun to use and useful to understand research networks. It can be used to discover new interesting related authors and their work. A second, more elaborate study is used to understand the relationship between the visualization approach and the type of insight that is gained.

\section{REFERENCES}

1. C. Chen and L. Carr. Trailblazing the literature of hypertext: author co-citation analysis (1989 - 1998). In Hypertext '99, 1999.

2. J. Heer and D. Boyd. Vizster: visualizing online social networks. Infovis 2005, pages 32-39, 2005.

3. I. Herman and G. e. a. Melancon. Graph visualization and navigation in information visualization: A survey. IEEE TVCG, 6(1):24-43, 2000.

4. G. Marchionini. From finding to understanding. ACM, 49(4):41-46.

5. M. Meire, X. Ochoa, and E. Duval. SAmgI: Automatic metadata generation v2.0. In Proc of Edmedia, pages 1195-1204, 2007.

6. T. Nagel and E. Duval. Muse: Visualizing the origins and connections of institutions based on co-authorship of publications. In Proc of 2nd Int. Workshop on Research 2.0, CEUR-WS.org vol 675 p 48-52, 2010.

7. G. Rossi. Improving Web information systems with navigational patterns. Computer Networks, 31(1116):1667-1678, 1999.

8. D. M. Russell and M. J. Stefik et al. The cost structure of sensemaking. In $C H I$ '93, pages 269-276, 1993.

9. P. Shannon, A. Markiel, and O. Ozier et al. Cytoscape: software environment for integrated models of biomolecular interaction networks. Genome Research, 13:2498-2504, 2003.

10. B. Shneiderman. The eyes have it: a task by data type taxonomy for information visualizations. Proc IEEE Symposium on Vis. Lang., pages 336-343, 1996.

11. M. Sorrows and S. Hirtle. The nature of landmarks for real and electronic spaces. Spatial information theory. Cognitive and computational foundations of geographic information science, pages 748-748, 1999. 